\section{Measuring surface tension on site}

The advantages of the BP50 measuring device supplied by

Krüss include the fact that it does not need a network connection and is easy to use. Potential applications include measuring the concentration of cleaning agents on site.

Concentrations above the critical micelle concentration (CMC), which are typical of cleaning baths, cannot be distinguished using static measurements of the surface tension. In contrast, the dynamic bubble pressure method used by the mobile measuring device is sensitive to changes in concentration above the CMC. For high-speed processes, such as printing, spraying or coating, it is the dynamic surface tension in the short-term that is the decisive factor. The dynamic bubble pressure technique of the instrument detects the surface tension which occurs just after surface formation at surface ages between 15 and 16,000 ms.

The measuring system, which consists of capillaries, an air supply, a pressure sensor and a temperature sensor, is contained in a mobile hand-held instrument. Disposable plastic capillaries mean that the hydrophobising process needed for glass capillaries can be dispensed with. The instrument works almost independently of the capillary immersion depth, which is an obvious benefit in the case of rapid tests. Water or other solvents can be used for calibration at different temperatures.

The measurements are defined and the instrument controlled by the BP50 add-in for LabDesk, the tensiometer software package. Various measuring programmes can be used to record timedependent surface tension or the measurements at a constant surface age. In addition, it is possible to determine whether a measurement is valid or invalid for the rapid quality test.

For more information: Krüss GmbH, D-22453 Hamburg, Germany, Tel.: +49 (0)40 514401 0, info@kruss.de, www.kruss.de

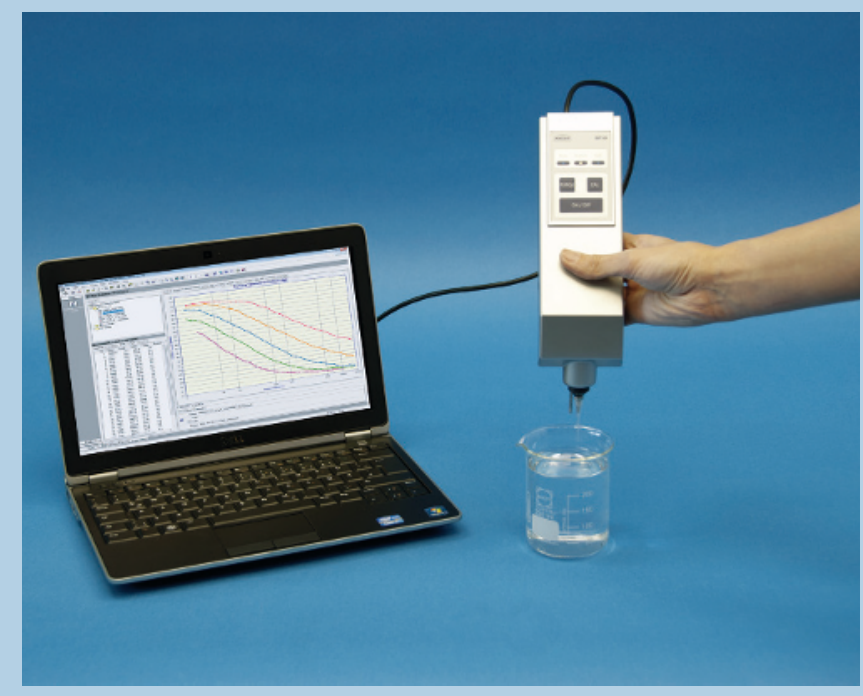

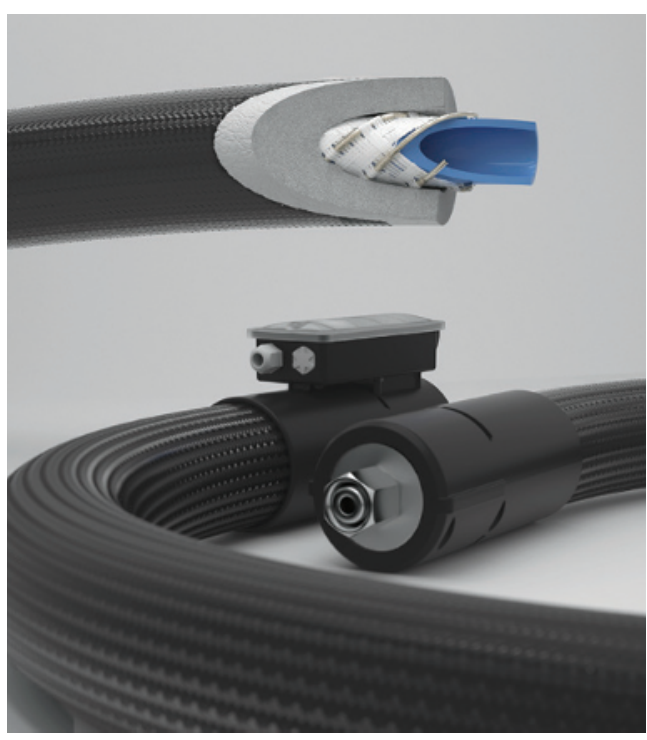

\section{Constant fluid temperature}

The performance of the Templine heated ho-

se system developed by Masterflex has been recognised in the vote held by the "CAV Chemie Anlagen und Verfahren" magazine to find the top product of 2011. The Templine system was awarded 3rd place in the contest. Because of its special design, it offers significant benefits when compared with conventional systems, which generally have one tape or cable that provides the heat. In a Templine heated hose, the internal hose that transports the fluid is completely surrounded by four heating cables which are held in place by heat-resistant braiding to ensure the even distribution of heat around the internal hose. The very close contact between the heating cables and the surface of the hose allows the heat to be transmitted directly. As a result, the heat transfer is around 30\% more efficient than in conventional systems.

The electrically heated hoses are ideal for a variety of fluids, gases and powders which have to be conveyed at a constant temperature, for example to maintain their flowability, as is the case when processing adhesives.

In the same way as for all Masterflex products, a variety of different hose connections is available for the heated hose system, including connectors made from brass, steel and stainless steel.

For more information: Masterflex AG,

D-45891 Gelsenkirchen, Germany,

Tel. +49 (0)20997077 0, info@masterflex.de 